\title{
Activated c-Jun N-Terminal Kinase Is Required for Axon Formation
}

\author{
Anthony A. Oliva Jr, ${ }^{1}$ Coleen M. Atkins, ${ }^{2}$ Lily Copenagle, ${ }^{1}$ and Gary A. Banker ${ }^{1}$ \\ ${ }^{1}$ Center for Research on Occupational and Environmental Toxicology and ${ }^{2}$ The Vollum Institute, Oregon Health \& Science University, Portland, Oregon \\ 97239
}

A critical transition in neuron development is formation of the axon, which establishes the polarized structure of the neuron that underlies its entire input and output capabilities. The morphological events that occur during axonogenesis have long been known, yet the molecular determinants underlying axonogenesis remain poorly understood. We demonstrate here that axonogenesis requires activated c-Jun N-terminal kinase (JNK). JNK is expressed throughout the neuron, but its phosphorylated, activated form is highly enriched in the axon. In young axons, activated JNK forms a proximodistal gradient of increasing intensity, beginning at about the point where the axon exceeds the lengths of the other neurites (minor processes). Treatment with SP600125, a specific inhibitor of JNK, reversibly inhibits axonogenesis but does not prevent the formation of minor processes or their differentiation into dendrites (based on their immunostaining with marker proteins). Expression of a dominant-negative construct against JNK similarly prevents axonogenesis. Investigation of JNK targets revealed that activating transcription factor- 2 is phosphorylated under normal conditions in neurons, and its phosphorylation is significantly attenuated after JNK inhibition. These results demonstrate that activated JNK is required for axonogenesis but not formation of minor processes or development of dendrites.

Key words: axon formation; axonogenesis; c-Jun N-terminal kinase; JNK; ATF-2; JIP-1; axon; SP600125

\section{Introduction}

Nearly every aspect of neuronal signaling depends on the development and maintenance of neuronal polarity (Craig and Banker, 1994). Neuronal polarity develops in a highly stereotypical manner that has been most extensively studied using cultured embryonic hippocampal neurons (Dotti et al., 1988). After initially forming a flattened, perisomatic lamellipodium (developmental stage 1), the neurons extend several short neurites (minor processes) that appear identical with respect to their morphology and molecular composition (stage 2). The first evidence of polarity occurs when one neurite undergoes an extended period of growth, becoming the axon (stage 3 ). Subsequently, the remaining neurites become dendrites (stage 4). The transition from stage 2 to stage 3 (axon formation, i.e., axonogenesis) is a defining event in establishing the highly polarized structure of the neuron. Although signaling pathways that play a role in neuronal specification have begun to be identified (Arimura and Kaibuchi, 2005; Jiang and Rao, 2005; Wiggin et al., 2005), much remains to be discovered.

We set out to screen for protein kinases that might be involved

Received Jan. 17, 2006; revised July 26, 2006; accepted July 28, 2006.

This work was supported by National Institutes of Health Grants NS17112, EY005285, and HD039884 and by the Nanobiotechnology Center, a Science and Technology Center Program of the National Science Foundation under Agreement ECS-9876771. We thank Barbara Smoody and Julie Luisi-Harp for technical assistance and Drs. John Bixby, Vance Lemmon, Bruce Magun, and Phil Stork for their critical suggestions.

Correspondence should be addressed to Dr. Anthony A. Oliva Jr, The Miami Project to Cure Paralysis, The University of Miami, Lois Pope LIFE Center (R-48), 1095 Northwest 14th Terrace, Miami, FL 33136. E-mail: aoliva@miamiproject.med.miami.edu.

D01:10.1523/JNEUROSCI.2625-06.2006

Copyright $\odot 2006$ Society for Neuroscience $\quad$ 0270-6474/06/269462-09\$15.00/0 in axonogenesis, and hypothesized the following: (1) such protein kinases would be preferentially localized to, or activated in, the axon and (2) inhibition of such kinases would selectively prevent axon formation, but not neurite formation in general. We report here that c-Jun N-terminal kinase (JNK) fulfills these criteria. JNK is a member of the mitogen-activated protein (MAP) kinase family (Minden and Karin, 1997; Harper and LoGrasso, 2001; Manning and Davis, 2003). More precisely, JNK comprises a MAP kinase subfamily encoded by three different genes (Jnk1, Jnk2, and Jnk3) that yield at least 10 different splice variants. All variants share a common epitope that, when dually phosphorylated at Thr183/Tyr185 (for JNK1), results in JNK becoming an active kinase [phosphorylated JNK (phospho-JNK)]. After phosphorylation and activation, JNK then phosphorylates a variety of downstream targets, such as activating transcription factor-2 (ATF-2).

Because of its robust activation during cellular stress, JNK has been studied extensively as a stress-activated protein kinase (thus its alternative name, stress-activated protein kinase). However, it is clear that JNK plays other important roles in neural functions. JNK signaling has been implicated in the development of cerebellar granule neurons (Coffey et al., 2000; Björkblom et al., 2005), and mice null for the $J n k 1$ gene exhibit abnormalities in axonal tracts (Chang et al., 2003). Mice dually null for Jnk1 and Jnk2 exhibit severe neurological defects and die during embryogenesis (Sabapathy et al., 1999). JNK has also been implicated in regulating the transcriptional events that regulate neurite outgrowth in PC12 cells (Yao et al., 1997; Kita et al., 1998) and axon regeneration in dorsal root ganglion neurons (Kenney and Kocsis, 1998).

Here, we demonstrate a new and unexpected role for JNK in 
regulating the development of neuronal polarity. We show that phospho-JNK is selectively enriched in both nascent and mature axons. Inhibiting JNK activation using pharmacological or molecular approaches blocks axonogenesis but does not inhibit neurite formation in general or prevent dendritic differentiation.

\section{Materials and Methods}

Neuronal cultures. Embryonic day 18 rat hippocampal neurons were plated onto coverslips in plating medium for $4 \mathrm{~h}$, followed by transfer of the coverslips to Neurobasal or N2.1 culturing medium, as described previously (Banker and Goslin, 1998). The culturing medium contained either $0 \mu \mathrm{M}$ (control) or $10 \mu \mathrm{M}$ SP600125 (Tocris Cookson, Ellisville, $\mathrm{MO}$ ). Either the N2.1 medium was conditioned with plated glia for at least $24 \mathrm{~h}$ in advance, followed by transfer of the medium to new dishes to make the cultures glia-cell free, or neurons were cultured with a glial feeder layer using the "sandwich" technique. All three culturing conditions yielded similar results. Transfections were performed using the Nucleofector electroporation system (Amaxa Biosystems, Gaithersburg, $\mathrm{MD)}$.

Plasmids. The transgene for the yellow fluorescent protein-JNKbinding domain (YFP-JBD) was constructed by linking the open reading for YFP $5^{\prime}$ in frame to the cDNA for the JBD (amino acid residues 127-281) of murine JNK interacting protein-1 (JIP-1).

Immunofluorescence and image analysis. Anti-phospho-JNK (9255; Cell Signaling Technology, Danvers, MA), anti-total JNK (610627; BD Transduction Laboratories, San Jose, CA), anti-phospho-ATF-2 (9225; Cell Signaling Technology), tau-1 (Boehringer Mannheim, Indianapolis, IN), and anti-MAP2 (ab5392; Abcam, Cambridge, MA) antibodies were used at 1:100, 1:200, 1:200, 1:10, and 1:30,000 dilution, respectively. Secondary antibodies were Alexa 350-, 488-, or 546-labeled goat anti-mouse or anti-rabbit (Invitrogen, Carlsbad, CA) and FITC-labeled goat antichicken (Abcam). All images were obtained within the linear range of a cooled CCD camera (MicroMax $5 \mathrm{MHz}$; Princeton Instruments, Trenton, $\mathrm{NJ}$ ).

Line-scans ( 5 pixels wide) were performed along the axon and minor processes, and the phospho-JNK/YFP ratio was calculated for each point. For each neuron, the average phospho-JNK/YFP intensity along all the minor processes was normalized to 1.0 , and all data were plotted on the basis of this normalization. Each plotted point represents a $1 \mu \mathrm{m}$ average of phospho-JNK/YFP along the neurites.

In Figure $2 f$, images of 21 random fields for each condition were taken from duplicate coverslips of three separate culture preparations, and all neurons that were completely in each field were used for analysis. An "axon" was defined as a neurite that was $\geq 45 \mu \mathrm{m}$ long and $\geq 10 \mu \mathrm{m}$ longer than all of the other neurites, on the basis of previously described criteria (Goslin and Banker, 1989; Esch et al., 1999).

For Figures 3 and 7, images from random fields for each condition were taken from two or three independent experiments, respectively. To quantify the lengths of all processes, the neurons were electroporated before plating with a construct encoding soluble green fluorescent protein, YFP-JBP, or cyan fluorescent protein (CFP) and grown on coverslips seeded with untransfected neurons. Process lengths for each neuron were measured using the National Institutes of Health NeuronJ neurite tracing program developed by Erik Meijering (http://imagescience.bigr.nl/meijering/software/neuronj/); all transgene-expressing neurons that were completely within each field were used for analysis. Cells electroporated with YFP-JBD that took on a non-neuronal appearance or appeared unhealthy were excluded from additional analysis.

For comparative tau-1 and MAP2 immunostaining, neurons were from the same culture preparations, grown in parallel under the respective conditions, and immunoprocessed in parallel under identical conditions. Images were obtained using the same exposure settings and were scaled identically. Similar parameters apply for images of Figure 5 and supplemental Figure 1 (available at www.jneurosci.org as supplemental material).

Neurons chronically cultured in the presence of SP600125 for 4 or $7 \mathrm{~d}$ were either grown on coverslips after transfection with soluble tomato autofluorescent protein (Shaner et al., 2004) and co-plated with untrans- fected neurons or grown in 96-well plates. Washouts were done via transfer to drug-free medium or medium replacement. Neurons were immunostained for tau-1 and MAP2, and the nuclei were stained with Hoechst 33258. Cell survival was assessed by comparing the number of Hoechst 33258-positive nuclei between control and SP600125-treated cultures, which were prepared via equal distributions from the same neuronal suspensions over two independent cultures. Neurons grown on coverslips were analyzed using NeuronJ as above; those grown in 96-well plates were analyzed using a KineticScan HCS Reader with the accompanying vHSC:Scan and vHCS:View software (Cellomics, Pittsburgh, PA).

Western blot analysis. Western blots were processed using antibodies against phospho-JNK (1:1000 dilution), then stripped and processed with anti-total JNK antibodies (1:1000 dilution), and then stripped and processed with anti- $\beta$-tubulin antibodies (1:5000 dilution) (Developmental Studies Hybridoma Bank, University of Iowa, Iowa City, IA). The $46 \mathrm{kDa}$ band was used for quantification, but the $54 \mathrm{kDa}$ band yielded similar results. Other Western blots were performed similarly using antiphospho-ATF-2 (9225; Cell Signaling Technology) and anti-total ATF-2 (9222; Cell Signaling Technology) antibodies at 1:1000 dilution. For the 90 min treatments with SP600125, cultures were transferred from control to SP600125-containing medium $22.5 \mathrm{~h}$ after plating.

Statistical analysis. Statistical analyses used were one-way ANOVA. Significance was determined using the Tukey's post hoc $t$ test or the unpaired Student's $t$ test. All data presented are mean \pm SEM.

\section{Results}

\section{Activated JNK is localized to mature and nascent axons}

During a screen for kinases that might be involved in axonogenesis, immunofluorescence microscopy revealed that phosphoJNK was preferentially enriched in the axons of mature (stage 4) cultured hippocampal neurons (Fig. 1a). At developmental stage 3 , shortly after axon formation, phospho-JNK was already enriched in the axon, where it was expressed in a distally directed gradient of increasing intensity, extending into the growth cone (Fig. 1b). To examine this axonal enrichment in more detail, we quantified the distribution of phospho-JNK. Before plating, neurons were electroporated with an expression plasmid encoding YFP to provide a cytoplasmic fill of the entire neuron, then immunostained after $1-2 \mathrm{~d}$ in culture. Line-scans were then traced along the imaged axons and minor processes to quantify the fluorescence. To correct for variations in neurite diameter (which often increases in the distal axon at this developmental stage), the ratio of phospho-JNK to YFP (phospho-JNK/YFP) was calculated at each point. In the representative stage 3 neuron analyzed in Figure $1 b$, phospho-JNK/YFP increased in a proximodistal gradient along the axon, increasing $\sim 7$ times above the average intensity of the minor processes (Fig. 1c). Interestingly, the increase in phospho-JNK began where the axon surpassed the length of the minor processes: this correlates well to the axon "critical length," which is the length that a neurite must surpass before it unequivocally can be identified as an axon (Esch et al., 1999), as well as the shortest length that the axon can be severed at (axotomized) and still retain its identity as an axon (Dotti and Banker, 1987). In stage 3 neurons with well established axons (>90 $\mu \mathrm{m}$ in length), phospho-JNK/YFP levels in the distal $10 \mu \mathrm{m}$ of the axon averaged $6.0 \pm 0.6$ times greater than the average in the minor processes $(n=10)$ and reached a maximum fold increase of $13.2 \pm 2.3$ along the axons. In contrast to phospho-JNK, total JNK (independent of phosphorylation state) had a more uniform distribution throughout all neurites (Fig. 1d,e).

In most stage 2 neurons, phospho-JNK staining was absent from neurites (Fig. $1 f$ ). However, in $\sim 3 \%$ of stage 2 neurons, phospho-JNK was enriched in the distal shaft and growth cone of a single neurite (Fig. $1 g$ ) (7 of 198 stage 2 neurons examined). 

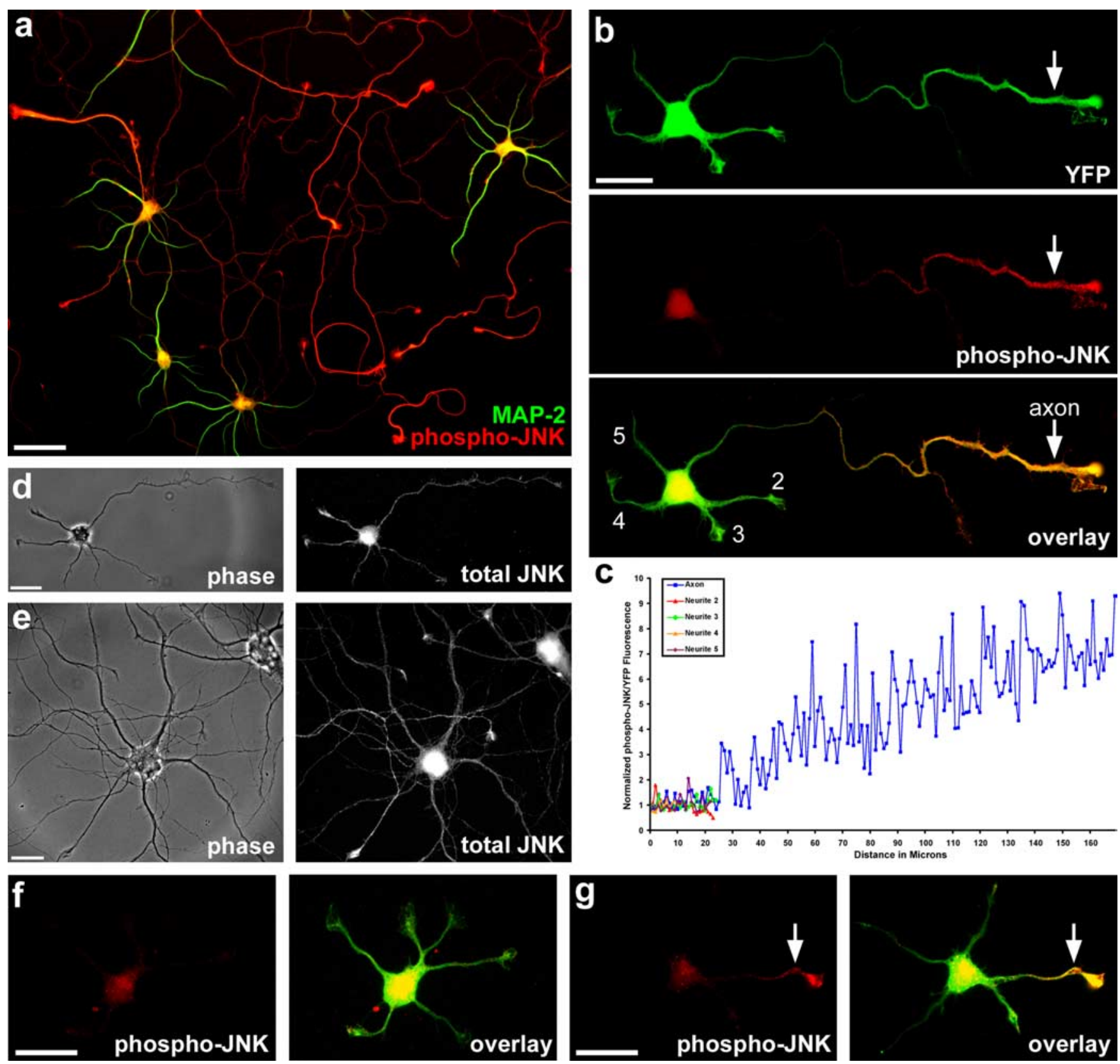

Figure 1. Phospho-JNK is enriched in axons. $\boldsymbol{a}$, After 1 week in culture, phospho-JNK immunoreactivity (red) was concentrated in the axons of hippocampal neurons, but essentially absent from dendrites (labeled with antibodies against MAP2; green). $\boldsymbol{b}$, A representative stage 3 neuron (24 $\mathrm{h}$ in culture) showing that phospho-JNK (red) was already enriched in the axon. c, Quantitative analysis revealed a pronounced proximodistal gradient of phospho-JNK in the neuron shown in $\boldsymbol{b}$. Changes in cell volume were corrected using expressed YFP as a cytoplasmic marker (green). $\boldsymbol{d}, \boldsymbol{e}$, Immunoreactivity for total JNK protein (regardless of phosphorylation state) was present throughout neurons at developmental stages 3 and 4 . $\boldsymbol{f}$, $\boldsymbol{g}$, Representative stage 2 neurons transfected to express soluble YFP. Although most stage 2 neurons showed an absence of phospho-JNK in all processes $(\boldsymbol{f})$, some had phospho-JNK enriched in the shaft and growth cone of a single neurite $(\boldsymbol{g}$, arrow). Scale bars: $\boldsymbol{a}, 50 \mu \mathrm{m} ; \boldsymbol{b}, \boldsymbol{d}, \boldsymbol{e}, 25 \mu \mathrm{m} ; \boldsymbol{f}, \boldsymbol{g}, 20 \mu \mathrm{m}$.

This suggests that JNK activation may be an early event during axonogenesis.

Pharmacological inhibition of JNK reversibly prevents axonogenesis but not neurite formation

Given the enrichment of phospho-JNK in developing axons, we investigated whether activated JNK is required for axonogenesis. SP600125 (JNK inhibitor II) is a potent, cell-permeable antagonist of JNK; at $10 \mu \mathrm{M}$, inhibition of isoforms from all three Jnk genes is 90-99\% (Bennett et al., 2001; Han et al., 2001). We cultured neurons in the presence or absence of $10 \mu \mathrm{M}$ SP600125 (Fig. $2 a-d$ ). In control cultures maintained for 24 or 48 h, $57.3 \pm$
$3.1 \%$ ( $n=253$ neurons over three independent experiments $)$ and $86.5 \pm 2.1 \%(n=272)$ of neurons, respectively, formed axons (Fig. 2f). Chronic SP600125 treatment (begun $4 \mathrm{~h}$ after plating before any neurites had formed) effectively eliminated axonogenesis: only $1.0 \pm 0.6 \%(n=312)$ and $2.4 \pm 0.9 \%(n=312)$ of neurons had axons at 24 and $48 \mathrm{~h}$, respectively (Fig. $2 f$ ). Inhibition of JNK did not prevent the formation of minor neurites.

To quantify these results, we determined the length of each neurite, ranked from longest to shortest (Fig. 3). For control cells at $2 \mathrm{~d}$ in culture, the longest neurite (the axon, in the case of neurons that had formed an axon) averaged $91.7 \pm 8.6 \mu \mathrm{m}$ in length, whereas the remaining neurites (i.e., the minor processes) 

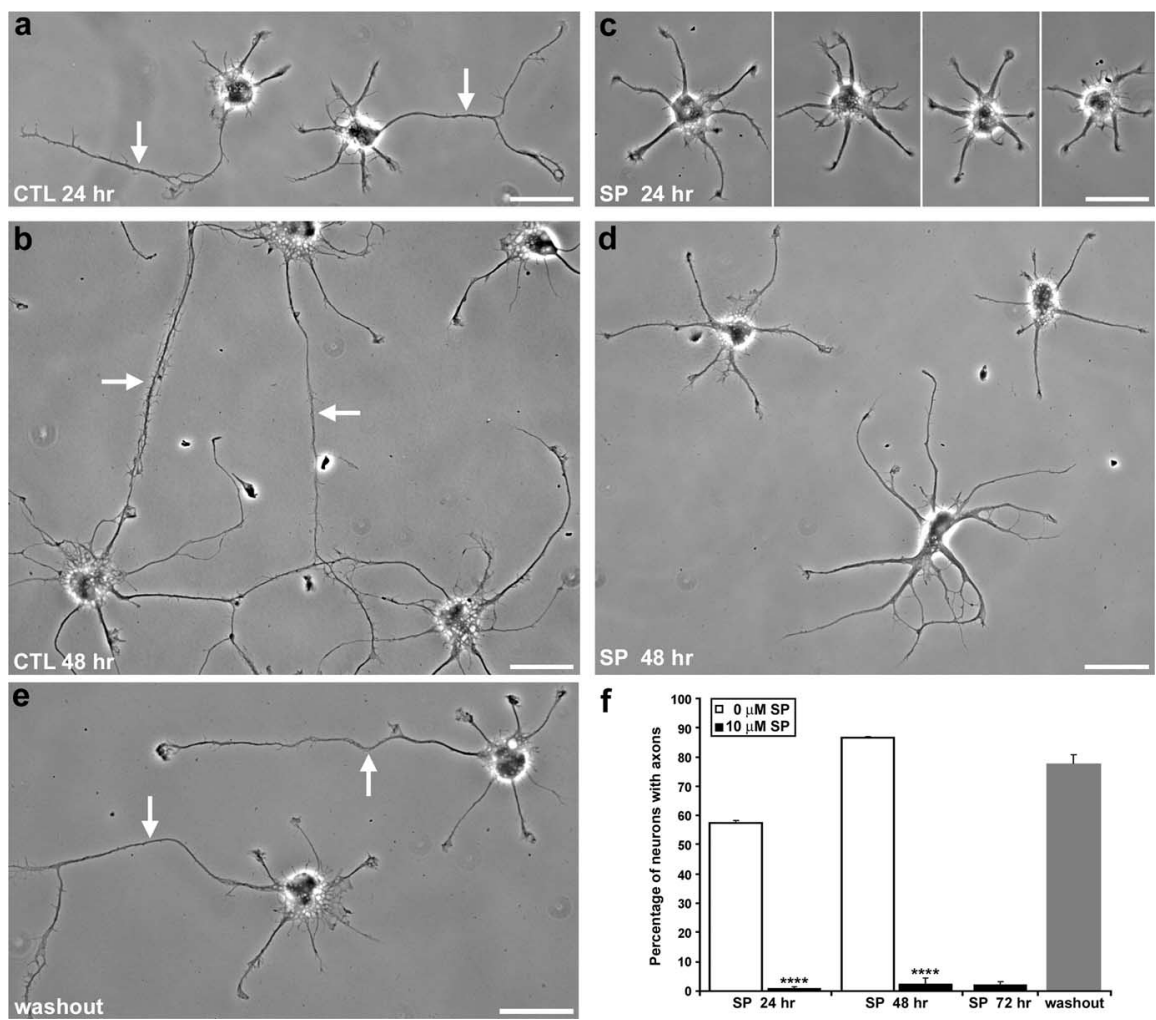

Figure 2. Axon formation was reversibly blocked by JNK inhibition. $\boldsymbol{a}, \boldsymbol{b}$, Control (CTL) neurons at 24 and $48 \mathrm{~h}$ in culture. $\boldsymbol{c}, \boldsymbol{d}$ Neurons chronically treated with the JNK inhibitor SP600125. $e$, Neurons incubated with SP600125 for $48 \mathrm{~h}$, followed by washout for 24 h.f, Quantification of axon-bearing neurons under control conditions (white bars), after chronic SP600125 treatment (black bars), or with SP600125 washout (gray bar). Axons from individual cells could not be accurately quantified in $72 \mathrm{~h}$ control cultures, because the cultures had formed too dense of a network. Error bars indicate SEM. ${ }^{* * *} p<0.0001$. Scale bars, $25 \mu \mathrm{m}$. SP, SP600125.

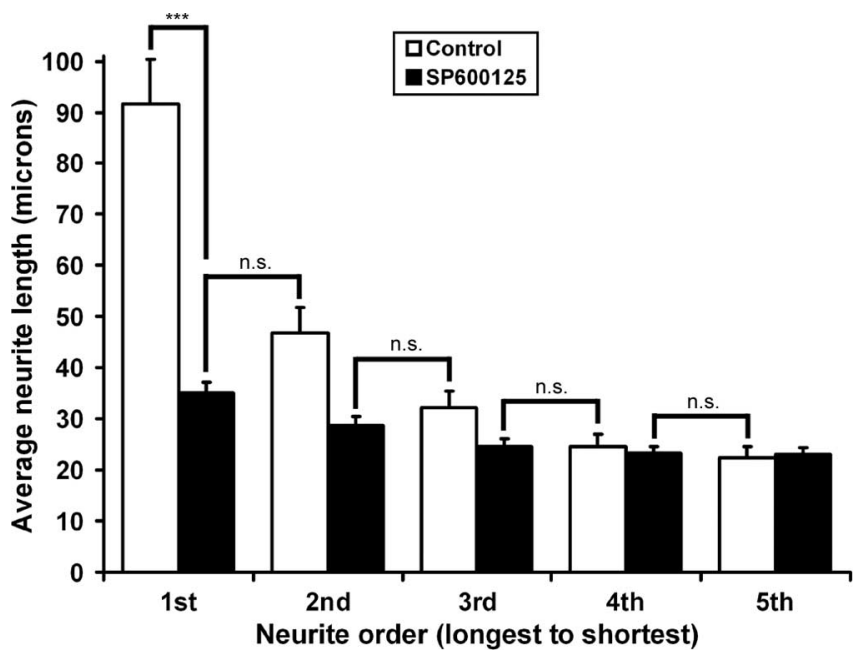

Figure 3. JNK inhibition does not affect the development of minor processes. Average neurite lengths at $48 \mathrm{~h}$ in culture under control conditions (open bars; $n=63$ neurons, 350 neurites) or with SP600125 treatment (filled bars; $n=108$ neurons, 752 neurites) are shown. ${ }^{* * *} p<0.001$. n.S., No statistical significance. Error bars indicate SEM.

were much shorter, averaging $32.6 \pm 2.0 \mu \mathrm{m}$ long $(n=65$ neurons). Neurons chronically treated with SP600125 did not develop a single neurite that was far longer than the rest (i.e., all of the neurites remained as minor processes). The average neurite length was $27.2 \pm 0.77 \mu \mathrm{m}$ long ( $n=107$ neurons), similar to the length of minor processes in control cells. Statistical analyses re- vealed no significant difference between the longest neurite in cells treated with SP60012 and the longest minor process (i.e., second longest neurite) of control neurons. Likewise, the average length of the second-longest neurite of neurons treated with SP600125 was not significantly different than that of the second longest minor process of control cells, etc. (Fig. 3). These results suggest that the JNK inhibitor did not suppress neurite growth, but rather selectively inhibited axon outgrowth.

The neurites that formed in the presence of SP600125 resembled dendrites in their branching and taper. These processes immunostained for the dendritic marker, MAP2, but not for the axonal marker, tau-1 (Fig. 4a,b). These results confirm that JNK inhibition selectively blocked axonogenesis. The blockade of axonogenesis by SP600125 was reversible (Fig. 2e,f). When neurons were treated with SP600125 for $48 \mathrm{~h}$, then placed in control medium for $24 \mathrm{~h}, 77.6 \pm 3.1 \%$ formed axons ( $n=202$ neurons).

To determine whether JNK inhibition prevents axon formation or merely delays axonogenesis, cultures were chronically treated with inhibitor for extended periods. After development in inhibitor for $4 \mathrm{~d}$, only $8.0 \pm 1.2 \%$ of neurons formed axons $(n=151)$, compared with $85.3 \pm$ $8.6 \%$ of control cells (Fig. $5 a$ ), as determined by tau- 1 immunoreactivity. After washout of inhibitor for $48 \mathrm{~h}, 87.4 \pm 6.8 \%$ of the neurons formed axons $(n=151 ; p<0.0001)$. Even after growth in inhibitor for $7 \mathrm{~d}$, only $6.8 \pm 1.7 \%$ of neurons formed axons $(n=849)$. Such prolonged treatments were deleterious, reducing cell survival by $50 \%$. Nevertheless, $24.8 \pm 0.9 \%$ of cells treated with inhibitor for $7 \mathrm{~d}$ formed axons $24 \mathrm{~h}$ after inhibitor removal ( $n=547$ cells). These results indicate that inhibition of JNK selectively and reversibly blocks axon outgrowth while allowing dendritic development to proceed.

\section{Inhibition of JNK activity strongly attenuates JNK phosphorylation}

Western blot analysis (Fig. 6a,b) confirmed that JNK was activated under basal conditions in developing neurons. Incubation of neurons with $10 \mu \mathrm{M}$ SP600125 for 24 h reduced phosphorylation of JNK to $11 \pm 2 \%(n=6)$ of control levels, whereas total JNK protein levels were not significantly affected. Even after incubation for only $90 \mathrm{~min}$, phospho-JNK was reduced to $22 \pm 2 \%$ $(n=6)$ by SP600125. Immunostaining confirmed that phosphoJNK was greatly reduced in neurons incubated with the JNK inhibitor for either $90 \mathrm{~min}$ or $24 \mathrm{~h}$ (supplemental Fig. 1, available at www.jneurosci.org as supplemental material). Because SP600125 is a competitive antagonist for the ATP-binding site in JNK, and appears not to block phosphorylation of JNK by its upstream activating kinases, these results imply that JNK undergoes positive-feedback activation or autophosphorylation within the axon, similar to previous observations in other cell types (Bennett et al., 2001). 

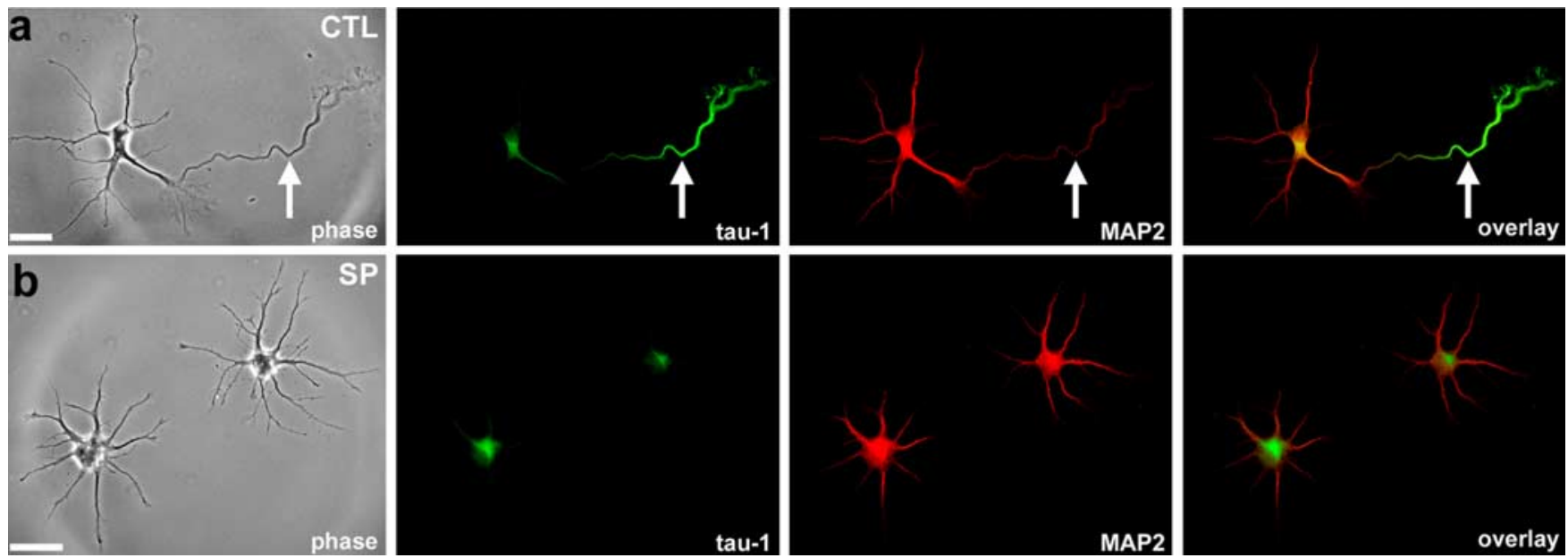

Figure 4. JNK inhibition results in neurites expressing the dendritic marker, MAP2, but not the axonal marker, tau-1. Control (CTL) neurons at $24 \mathrm{~h}$ in culture (a) or chronically SP600125 (SP)-treated neurons at $48 \mathrm{~h}$ in culture (b) revealed that SP600125-treated neurons had MAP2-positive, but tau-1-negative, neurites. Arrows denote axons. Scale bars, $25 \mu \mathrm{m}$.

\section{Dominant-negative inhibition of JNK} prevents axonogenesis

To verify our pharmacological results, we used a dominant-negative approach to inhibit JNK. JIP-1 is a scaffolding protein that brings together JNK and its upstream activators (Whitmarsh et al., 1998). Overexpression of the JBD portion of JIP-1 acts as an effective inhibitor of JNK (Dickens et al., 1997; Morrison and Davis, 2003). We created an expression vector encoding a fusion protein consisting of YFP $\mathrm{N}$-terminally linked to the JBD of JIP-1 (YFP-JBD). Before plating, neurons were either transfected with the YFP-JBD construct, or with a construct for soluble CFP (normal cell controls), and then the neurons were co-plated. At $2 \mathrm{~d}$ in culture, only a few of the YFP-JBD-expressing neurons had axons (Fig. 7). Morphologically, the YFP-JBD-expressing neurons were similar to those treated with SP600125. The processes of YFP-JBD neurons averaged in length from $\sim 10$ to $30 \mu \mathrm{m}$, slightly less than those of the minor processes of control neurons. Thus, as was the case with SP600125 treatment, molecular inhibition of JNK blocked axonogenesis but not neurite formation in general. Even 4 weeks after transfection, we observed neurons that had not formed axons but that did have well developed dendritic arbors (supplemental Fig. 2, available at www.jneurosci. org as supplemental material).

\section{JNK activation leads to ATF-2}

phosphorylation during development of neuronal polarity

Activated JNK can phosphorylate several targets, including ATF-2 (also known as CRE-BP1, CREB2, $\mathrm{mXBP}$ ). ATF-2 is a member of the ATF/CREB (cAMP response element-binding protein) family
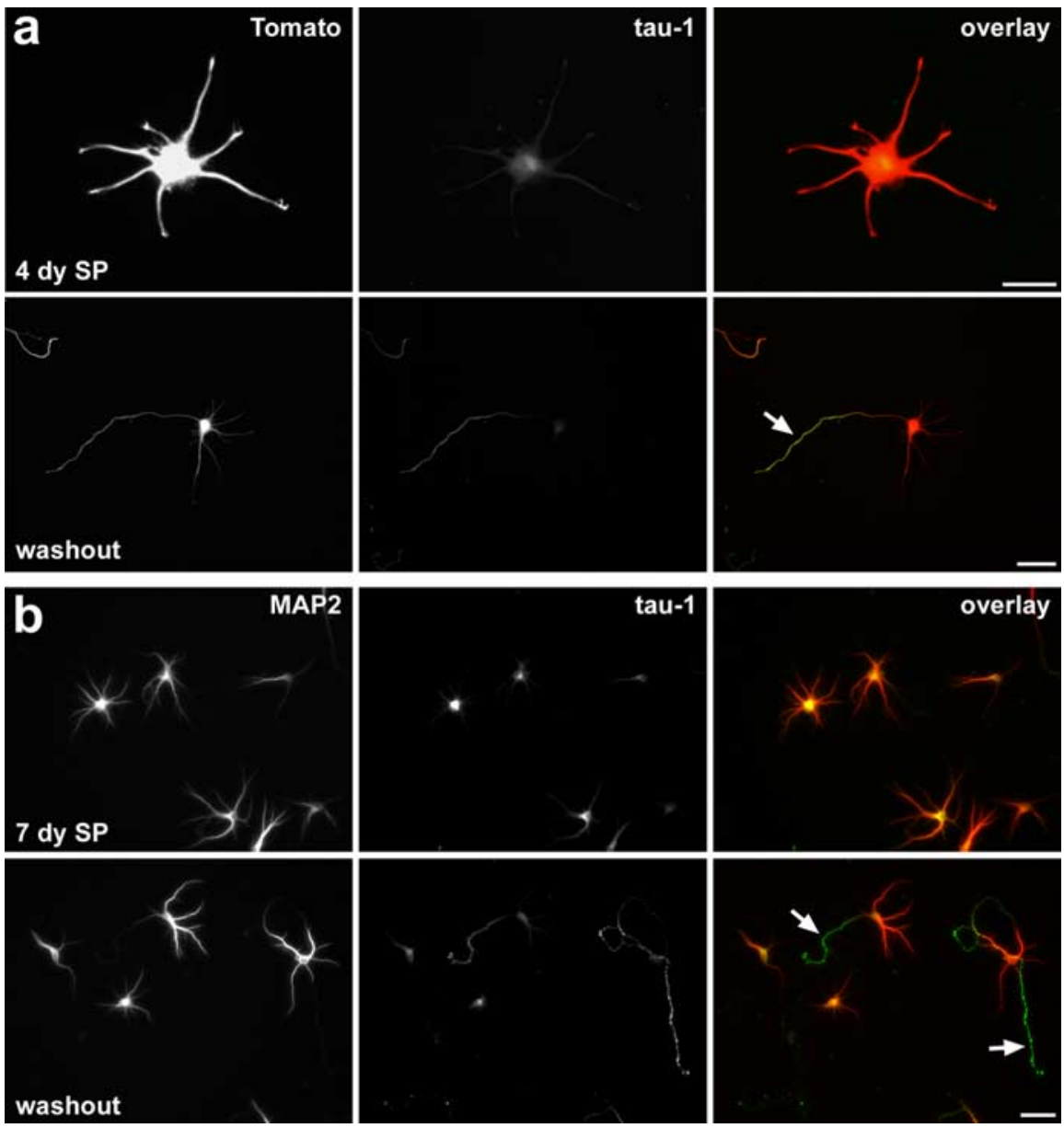

Figure 5. Long-term treatment with JNK inhibitor continues to reversibly prevent axon formation. $\boldsymbol{a}$, Neurons transfected to express soluble tomato fluorescent protein and immunostained for tau-1. Top panels, Representative neuron chronically treated with SP600125 for $4 \mathrm{~d}$ ( $4 \mathrm{dy}$ ), showing continued inhibition of axon formation. Bottom panels, Representative neuron cultured for $4 \mathrm{~d}$ in the chronic presence of SP600125, followed by washout for $24 \mathrm{~h}$. The neuron formed a single axon. $\boldsymbol{b}$, Neurons at $7 \mathrm{~d}(7 \mathrm{dy})$ in culture immunostained for tau- 1 and MAP2. Top panels, Representative neurons chronically treated with SP600125. The neurons did not form axons but had MAP2-immunopositive neurites that resembled dendrites. Bottom panels, Representative neurons cultured in the presence of SP600125 for $6 \mathrm{~d}$, followed by washout for $24 \mathrm{~h}$. Two of the four neurons formed axons (arrows). Scale bars: $\boldsymbol{a}, 25 \mu \mathrm{m}$ (top panels) and $50 \mu \mathrm{m}$ (bottom panels); $\boldsymbol{b}, 50 \mu \mathrm{m}$. SP, SP600125. 


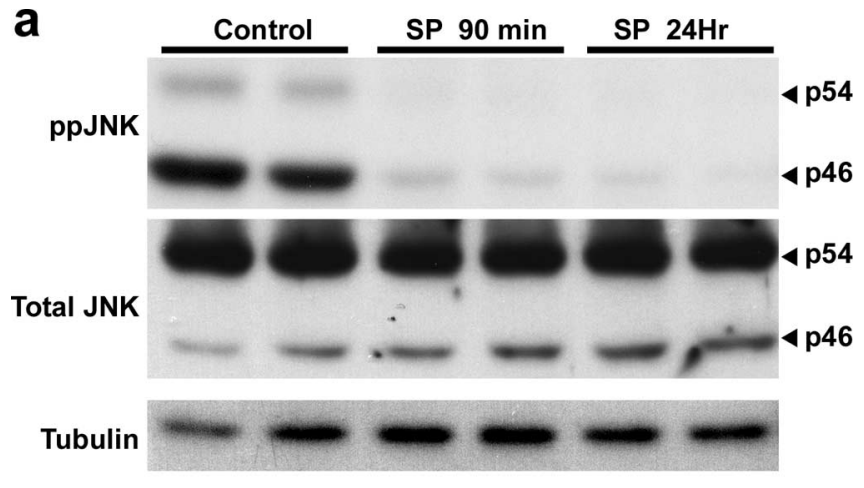

b

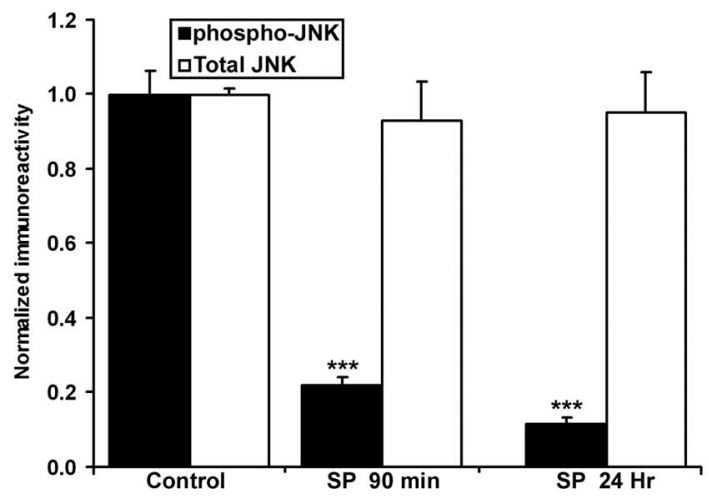

Figure 6. Phospho-JNK levels dramatically decreased with SP600125 treatment. $\boldsymbol{a}$, Representative Western blots of cultures $24 \mathrm{~h}$ after plating that were chronically treated with SP600125 (SP 24Hr), treated with SP600125 for 90 min just before lysing (SP $90 \mathrm{~min}$ ), or untreated (control). $\boldsymbol{b}$, Graph showing that chronic and acute treatment with SP600125 significantly inhibited phosphorylation of JNK but did not affect total JNK levels ( $n=6$ for each condition). ${ }^{* * *} p<0.001$. Error bars indicate SEM.

of transcription factors that bind to CRE (cAMP-responsive element) consensus sites and regulate numerous neuronal genes (Gupta et al., 1995, 1996; van Dam et al., 1995; Herdegen and Leah, 1998). Phosphorylated ATF-2 (phospho-ATF-2) was found to be enriched in the axon, similar to the enrichment of phospho-JNK (supplemental Fig. 3, available at www. jneurosci.org as supplemental material). To determine whether JNK activation leads to ATF-2 phosphorylation during the development of neuronal polarity, we treated neurons with SP600125. Chronic or acute treatment with SP600125 (the former of which inhibited axon formation) decreased phospho-ATF-2 (phosphorylated at T51/T53 in rat) by $65 \pm 3$ and $60 \pm 5 \%(n=6)$, respectively, but did not significantly affect total ATF-2 levels (Fig. 8). a
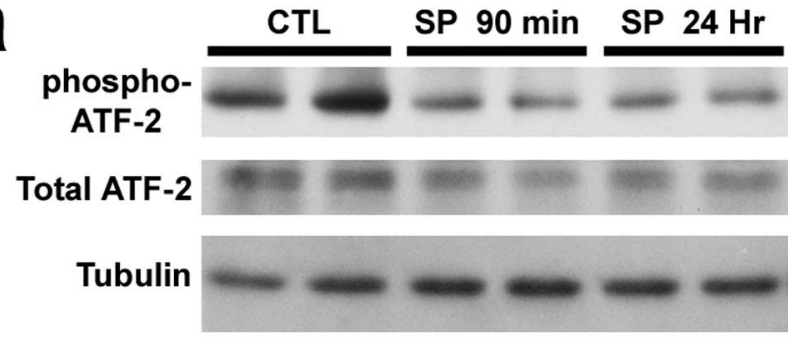

b

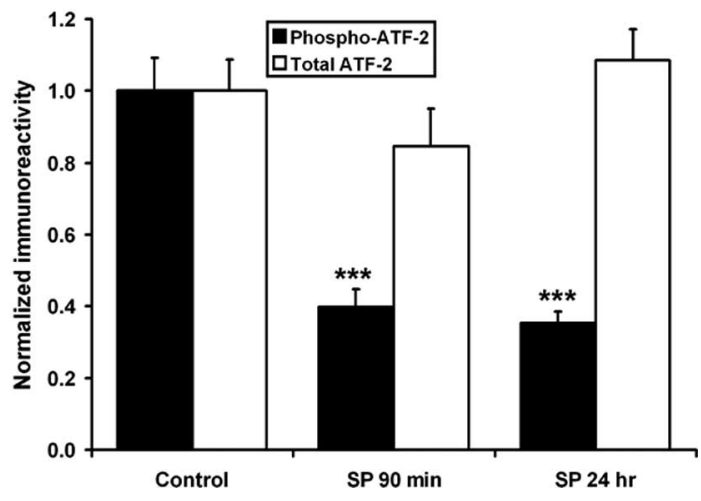

Figure 8. Phosphorylation of the JNK substrate ATF-2 is attenuated by SP600125 (SP). $\boldsymbol{a}$, Western blots demonstrating that ATF-2 was dually phosphorylated (at T51/T53) under basal conditions, which decreased with acute or chronic inhibition of JNK. CTL, Control. $\boldsymbol{b}$, Quantification revealed JNK inhibition significantly decreased ATF-2 phosphorylation ( $n=6$ for each condition). ${ }^{* * *} p<0.001$. Error bars indicate SEM.

\section{Discussion}

Our results demonstrate a novel role for the JNK family of kinases in the development of neuronal polarity. Whereas JNK is present throughout the cell, phospho-JNK is highly enriched in the axon, beginning early in stage 3 and persisting throughout development. Inhibiting JNK by treatment with specific pharmacological blockers or by expressing dominant-negative constructs did not prevent the formation of minor processes or their subsequent differentiation as dendrites but did effectively abolished axon formation. The pharmacological inhibition of axon formation can be maintained for at least 1 week, after which the cultures die; yet, inhibition is reversible while the cells are still living. When the inhibitor is removed, some cells remain capable of forming axons and become polarized. We do not know whether ectopic expression of activated JNK can result in the formation of multiple axons, which has been reported for other signaling molecules
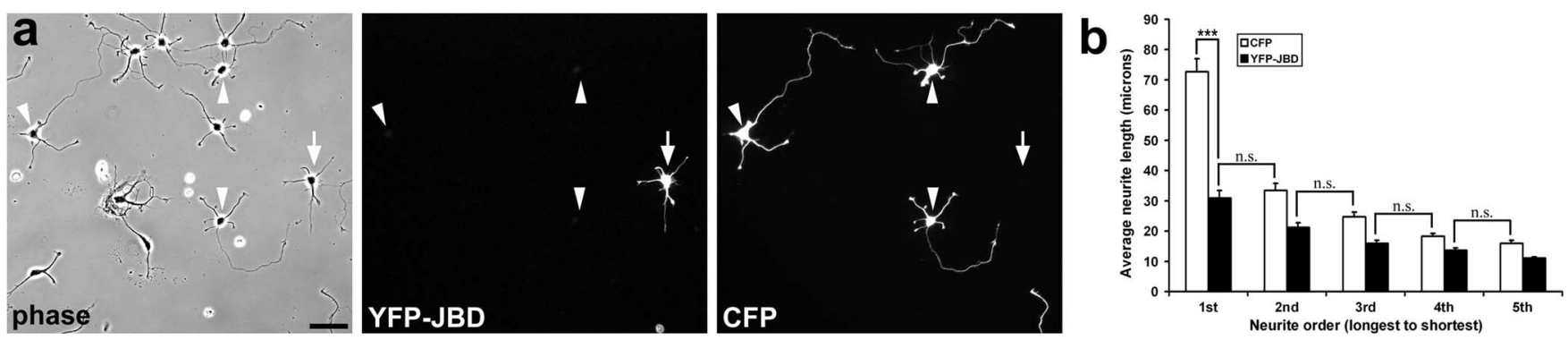

Figure 7. Molecular inhibition of JNK interferes with axonogenesis. $\boldsymbol{a}$, Representative neurons at $2 \mathrm{~d}$ in culture from a coculture of neurons separately transfected to express YFP-JBD (a dominant-negative inhibitor of JNK) or soluble (FP (controls). $\boldsymbol{b}$, Quantification of average neurite lengths for neurons either expressing YFP-JBD (filled bars; $n=91$ neurons, 520 processes over 3 independent experiments) or soluble (FP (open bars; $n=92$ neurons, 407 processes) at $2 \mathrm{~d}$ in culture. Scale bar, $50 \mu \mathrm{m}$. ${ }^{* *} p<0.001$. n.S., No statistical significance. Error bars indicate SEM. 
involved in the development of neuronal polarity such as CRMP2 and Rap1B (Inagaki et al., 2001; Schwamborn and Püschel, 2004). Such experiments will require identifying those JNK isoforms that mediate axon growth and developing appropriate constitutively active constructs, tasks that have so far proven difficult (Zheng et al., 1999).

Several aspects of our findings deserve additional comment. First, we found that phospho-JNK is present all along the axon, forming a proximodistal gradient of increasing activity at stage 3 that then becomes more uniformly distributed along the axon by stage 4. This resembles the localization of CRMP-2 but contrasts with that of other signaling molecules that have been implicated in the regulation of neuronal polarity, which are preferentially enriched in the growth cone (Arimura and Kaibuchi, 2005; Wiggin et al., 2005). The gradient of phospho-JNK begins $\sim 30-40$ $\mu \mathrm{m}$ from the cell body (Fig. 1c), which closely corresponds to the critical length for axon specification (Goslin and Banker, 1989). When the axon is transected proximal to the critical length, polarity is lost and a new axon forms from a different site; when transected beyond the critical length, the axon regenerates and polarity is maintained. Thus, transections that lead to a loss of neuronal polarity would eliminate the activated JNK present in the axon.

The degree to which JNK activation occurs preferentially in the axon is also striking. On average, the concentration of activated JNK is sevenfold higher in the distal axon than in the minor processes, and this selective activation of JNK can occur in stage 2 neurons. It has been suggested that JNK acts as a molecular switch, responding to a variety of upstream signals in an all-ornone manner (Bagowski and Ferrell, 2001; Ferrell, 2002). The degree to which JNK is selectively activated in the axon may reflect this feature of JNK signaling.

Finally, JNK phosphorylates a variety of nuclear and cytoplasmic targets, including transcription factors, microtubuleassociated proteins, and proteins that interact with the actin cytoskeleton (Reynolds et al., 2000; Chang et al., 2003; Yoshida et al., 2004; Björkblom et al., 2005). Obviously, regulation of the microtubule and actin cytoskeletons plays an integral role in channeling growth to the emerging axon, the defining event in the specification of neuronal polarity. Although specification of the axon does not require gene transcription (Jareb and Banker, 1997), axonogenesis is likely to lead to transcriptional changes to provide the materials necessary for axonal elongation and maintenance. ATF-2, activated via JNK, is a likely candidate for mediating these effects. Interestingly, after partial optic nerve crush, a high ATF-2 expression level in retinal ganglion cells correlates with neuronal survival (Kreutz et al., 1999). Similarly, after axotomy of retinal ganglion cells, ATF-2 appears to play a role in neuronal survival and regeneration (Robinson, 1996). Thus, JNK is uniquely placed to regulate axonal growth directly, via its effects on the cytoskeleton, and over the long term, by regulating transcription.

Other signaling molecules that regulate neuronal polarity have been identified. These include the protein kinases GSK3 $\beta$ (glycogen synthase kinase-3 $\beta$ ), phosphatidylinositol 3' (PI3) kinase, and SAD kinases (the mammalian homologs of the Caenorhabditis elegans protein SAD-1, for synapses of the amphid defective); small GTPases cdc42, rap1B, and ras; and associated scaffolding complexes (Shi et al., 2003; Schwamborn and Püschel, 2004; Jiang et al., 2005; Kishi et al., 2005; Yoshimura et al., 2005, 2006). In other cells types, JNK activation sometimes occurs downstream of Rap1 (Ling et al., 2003), cdc42 (Coso et al., 1995), or PI3 kinase (Logan et al., 1997); under other circum- stances, JNK and Rap1 independently act in parallel (Mochizuki et al., 2000). It will be important to determine how JNK interacts with these other signaling pathways during the development of neuronal polarity. But given that Rap1B, cdc42, and PI3 kinase activity (Shi et al., 2003; Schwamborn and Püschel, 2004) appear to localize at the axon tip (which strikingly contrasts to phosphoJNK, which is throughout most of the length of the axon), it seems unlikely that they activate JNK in the axon.

Besides JNK, members of another MAP kinase subfamily, p44/p42 extracellular signal-regulated kinase (ERK1/2), has also been implicated in neurite outgrowth and in the development of neuronal polarity. In PC12 cells, neurotrophin-induced neuritogenesis requires activation of the ERK pathway, as well as PI3 kinase and Akt (Vaudry et al., 2002; Xiao and Liu, 2003; Kim et al., 2004). In contrast to PC12 cells, inhibition of ERK1/2 with PD98059 [2-(2-amino-3-methoxy-phenyl)chromen-4-one] (Yoshimura et al., 2006) or U0126 [(2Z,3Z)-2,3-bis[amino-(2aminophenyl)sulfanyl-methylidene]butanedinitrile] (A. A. Oliva, V. P. Lemmon, and G. A. Banker, unpublished observations) does not block axon formation in cultured hippocampal neurons. However, overexpression of $\mathrm{H}$-Ras increases the proportion of neurons with two or more axons, and this effect is attenuated by PD98059 (Yoshimura et al., 2006). Identifying the upstream regulatory mechanisms for each protein kinase cascade may shed light on the relative contributions of these two pathways for the establishment and maintenance of neuronal polarity.

What might account for the selective activation of JNK in the axon, given that JNK appears uniformly distributed throughout the neuron? It may be that MKK4 or MKK7, the kinases that activate JNK, or the activators of these upstream kinases (Davis, 2000), are preferentially activated in the developing axon. JNK may also be preferentially complexed in the axon with JIP-1, which links JNK with its activating kinases (Morrison and Davis, 2003). This seems plausible because JIP-1 binds kinesin-1 (Verhey et al., 2001) and kinesin-1 translocates preferentially into the axon (Nakata and Hirokawa, 2003). Indeed, live-cell imaging reveals that the selective and persistent accumulation kinesin- 1 in a single neurite is among the earliest known molecular indicators of axonal specification (Jacobson et al., 2006). JNK can also phosphorylate proteins that regulate the microtubule and actin cytoskeleton (Reynolds et al., 2000; Chang et al., 2003; Yoshida et al., 2004; Björkblom et al., 2005) and control cell motility (Gdalyahu et al., 2004; Xia and Karin, 2004). It might also enhance the axonal transport of other kinesin-1 cargoes, such as CRMP-2 (Inagaki et al., 2001; Arimura et al., 2004; Kimura et al., 2005). If JNK modification of the axonal cytoskeleton enhances kinesin-1 translocation, this could contribute to the positive feedback thought to underlie axonogenesis (Goslin and Banker, 1989; Craig and Banker, 1994; Andersen and $\mathrm{Bi}, 2000)$.

\section{References}

Andersen SS, Bi GQ (2000) Axon formation: a molecular model for the generation of neuronal polarity. BioEssays 22:172-179.

Arimura N, Kaibuchi K (2005) Key regulators in neuronal polarity. Neuron 48:881-884.

Arimura N, Menager C, Fukata Y, Kaibuchi K (2004) Role of CRMP-2 in neuronal polarity. J Neurobiol 58:34-47.

Bagowski CP, Ferrell Jr JE (2001) Bistability in the JNK cascade. Curr Biol 11:1176-1182.

Banker GA, Goslin K (1998) Culturing nerve cells. Cambridge, MA: MIT.

Bennett BL, Sasaki DT, Murray BW, O’Leary EC, Sakata ST, Xu W, Leisten JC, 
Motiwala A, Pierce S, Satoh Y, Bhagwat SS, Manning AM, Anderson DW (2001) SP600125, an anthrapyrazolone inhibitor of Jun N-terminal kinase. Proc Natl Acad Sci USA 98:13681-13686.

Björkblom B, Östman N, Hongisto V, Komarovski V, Filen JJ, Nyman TA, Kallunki T, Courtney MJ, Coffey ET (2005) Constitutively active cytoplasmic c-Jun N-terminal kinase 1 is a dominant regulator of dendritic architecture: role of microtubule-associated protein 2 as an effector. J Neurosci 25:6350-6361.

Chang L, Jones Y, Ellisman MH, Goldstein LS, Karin M (2003) JNK1 is required for maintenance of neuronal microtubules and controls phosphorylation of microtubule-associated proteins. Dev Cell 4:521-533.

Coffey ET, Hongisto V, Dickens M, Davis RJ, Courtney MJ (2000) Dual roles for c-Jun $\mathrm{N}$-terminal kinase in developmental and stress responses in cerebellar granule neurons. J Neurosci 20:7602-7613.

Coso OA, Chiariello M, Yu JC, Teramoto H, Crespo P, Xu N, Miki T, Gutkind JS (1995) The small GTP-binding proteins Racl and Cdc42 regulate the activity of the JNK/SAPK signaling pathway. Cell 81:1137-1146.

Craig AM, Banker G (1994) Neuronal polarity. Annu Rev Neurosci $17: 267-310$

Davis RJ (2000) Signal transduction by the JNK group of MAP kinases. Cell 103:239-252.

Dickens M, Rogers JS, Cavanagh J, Raitano A, Xia Z, Halpern JR, Greenberg ME, Sawyers CL, Davis RJ (1997) A cytoplasmic inhibitor of the JNK signal transduction pathway. Science 277:693-696.

Dotti CG, Banker GA (1987) Experimentally induced alteration in the polarity of developing neurons. Nature 330:254-256.

Dotti CG, Sullivan CA, Banker GA (1988) The establishment of polarity by hippocampal neurons in culture. J Neurosci 8:1454-1468.

Esch T, Lemmon V, Banker G (1999) Local presentation of substrate molecules directs axon specification by cultured hippocampal neurons. J Neurosci 19:6417-6426.

Ferrell Jr JE (2002) Self-perpetuating states in signal transduction: positive feedback, double-negative feedback and bistability. Curr Opin Cell Biol 14:140-148.

Gdalyahu A, Ghosh I, Levy T, Sapir T, Sapoznik S, Fishler Y, Azoulai D, Reiner O (2004) DCX, a new mediator of the JNK pathway. EMBO J 23:823-832.

Goslin K, Banker G (1989) Experimental observations on the development of polarity by hippocampal neurons in culture. J Cell Biol 108:1507-1516.

Gupta S, Campbell D, Derijard B, Davis RJ (1995) Transcription factor ATF2 regulation by the JNK signal transduction pathway. Science 267:389-393.

Gupta S, Barrett T, Whitmarsh AJ, Cavanagh J, Sluss HK, Derijard B, Davis RJ (1996) Selective interaction of JNK protein kinase isoforms with transcription factors. EMBO J 15:2760-2770.

Han Z, Boyle DL, Chang L, Bennett B, Karin M, Yang L, Manning AM, Firestein GS (2001) c-Jun N-terminal kinase is required for metalloproteinase expression and joint destruction in inflammatory arthritis. J Clin Invest 108:73-81.

Harper SJ, LoGrasso P (2001) Signalling for survival and death in neurones: the role of stress-activated kinases, JNK and p38. Cell Signal 13:299-310.

Herdegen T, Leah JD (1998) Inducible and constitutive transcription factors in the mammalian nervous system: control of gene expression by Jun, Fos and Krox, and CREB/ATF proteins. Brain Res Brain Res Rev 28:370-490.

Inagaki N, Chihara K, Arimura N, Menager C, Kawano Y, Matsuo N, Nishimura T, Amano M, Kaibuchi K (2001) CRMP-2 induces axons in cultured hippocampal neurons. Nat Neurosci 4:781-782.

Jacobson C, Schnapp B, Banker G (2006) A change in the selective translocation of the kinesin-1 motor domain marks the initial specification of the axon. Neuron 49:797-804.

Jareb M, Banker G (1997) Inhibition of axonal growth by brefeldin A in hippocampal neurons in culture. J Neurosci 17:8955-8963.

Jiang H, Rao Y (2005) Axon formation: fate versus growth. Nat Neurosci 8:544-546.

Jiang H, Guo W, Liang X, Rao Y (2005) Both the establishment and the maintenance of neuronal polarity require active mechanisms: critical roles of GSK-3beta and its upstream regulators. Cell 120:123-135.

Kenney AM, Kocsis JD (1998) Peripheral axotomy induces long-term c-Jun amino-terminal kinase-1 activation and activator protein- 1 binding activity by c-Jun and junD in adult rat dorsal root ganglia in vivo. J Neurosci 18:1318-1328.

Kim Y, Seger R, Suresh Babu CV, Hwang SY, Yoo YS (2004) A positive role of the PI3-K/Akt signaling pathway in PC12 cell differentiation. Mol Cells 18:353-359.

Kimura T, Watanabe H, Iwamatsu A, Kaibuchi K (2005) Tubulin and CRMP-2 complex is transported via kinesin-1. J Neurochem 93:1371-1382.

Kishi M, Pan YA, Crump JG, Sanes JR (2005) Mammalian SAD kinases are required for neuronal polarization. Science 307:929-932.

Kita Y, Kimura KD, Kobayashi M, Ihara S, Kaibuchi K, Kuroda S, Ui M, Iba H, Konishi H, Kikkawa U, Nagata S, Fukui Y (1998) Microinjection of activated phosphatidylinositol-3 kinase induces process outgrowth in rat PC12 cells through the Rac-JNK signal transduction pathway. J Cell Sci 111:907-915.

Kreutz MR, Bien A, Vorwerk CK, Bockers TM, Seidenbecher CI, Tischmeyer W, Sabel BA (1999) Co-expression of c-Jun and ATF-2 characterizes the surviving retinal ganglion cells which maintain axonal connections after partial optic nerve injury. Brain Res Mol Brain Res 69:232-241.

Ling L, Zhu T, Lobie PE (2003) Src-CrkII-C3G-dependent activation of Rap1 switches growth hormone-stimulated p44/42 MAP kinase and JNK/ SAPK activities. J Biol Chem 278:27301-27311.

Logan SK, Falasca M, Hu P, Schlessinger J (1997) Phosphatidylinositol 3-kinase mediates epidermal growth factor-induced activation of the c-Jun N-terminal kinase signaling pathway. Mol Cell Biol 17:5784-5790.

Manning AM, Davis RJ (2003) Targeting JNK for therapeutic benefit: from junk to gold? Nat Rev Drug Discov 2:554-565.

Minden A, Karin M (1997) Regulation and function of the JNK subgroup of MAP kinases. Biochim Biophys Acta 1333:F85-F104.

Mochizuki N, Ohba Y, Kobayashi S, Otsuka N, Graybiel AM, Tanaka S, Matsuda M (2000) Crk activation of JNK via C3G and R-Ras. J Biol Chem 275:12667-12671.

Morrison DK, Davis RJ (2003) Regulation of MAP kinase signaling modules by scaffold proteins in mammals. Annu Rev Cell Dev Biol 19:91-118.

Nakata T, Hirokawa N (2003) Microtubules provide directional cues for polarized axonal transport through interaction with kinesin motor head. J Cell Biol 162:1045-1055.

Reynolds CH, Betts JC, Blackstock WP, Nebreda AR, Anderton BH (2000) Phosphorylation sites on tau identified by nanoelectrospray mass spectrometry: differences in vitro between the mitogen-activated protein kinases ERK2, c-Jun N-terminal kinase and P38, and glycogen synthase kinase-3beta. J Neurochem 74:1587-1595.

Robinson GA (1996) Changes in the expression of transcription factors ATF-2 and Fra- 2 after axotomy and during regeneration in rat retinal ganglion cells. Brain Res Mol Brain Res 41:57-64.

Sabapathy K, Jochum W, Hochedlinger K, Chang L, Karin M, Wagner EF (1999) Defective neural tube morphogenesis and altered apoptosis in the absence of both JNK1 and JNK2. Mech Dev 89:115-124.

Schwamborn JC, Püschel AW (2004) The sequential activity of the GTPases Rap1B and Cdc42 determines neuronal polarity. Nat Neurosci 7:923-929.

Shaner NC, Campbell RE, Steinbach PA, Giepmans BN, Palmer AE, Tsien RY (2004) Improved monomeric red, orange and yellow fluorescent proteins derived from Discosoma sp. red fluorescent protein. Nat Biotechnol 22:1567-1572.

Shi SH, Jan LY, Jan YN (2003) Hippocampal neuronal polarity specified by spatially localized mPar3/mPar6 and PI 3-kinase activity. Cell 112:63-75.

van Dam H, Wilhelm D, Herr I, Steffen A, Herrlich P, Angel P (1995) ATF-2 is preferentially activated by stress-activated protein kinases to mediate c-jun induction in response to genotoxic agents. EMBO J 14:1798-1811.

Vaudry D, Stork PJ, Lazarovici P, Eiden LE (2002) Signaling pathways for PC12 cell differentiation: making the right connections. Science 296:1648-1649.

Verhey KJ, Meyer D, Deehan R, Blenis J, Schnapp BJ, Rapoport TA, Margolis B (2001) Cargo of kinesin identified as JIP scaffolding proteins and associated signaling molecules. J Cell Biol 152:959-970.

Whitmarsh AJ, Cavanagh J, Tournier C, Yasuda J, Davis RJ (1998) A mam- 
malian scaffold complex that selectively mediates MAP kinase activation. Science 281:1671-1674.

Wiggin GR, Fawcett JP, Pawson T (2005) Polarity proteins in axon specification and synaptogenesis. Dev Cell 8:803-816.

Xia Y, Karin M (2004) The control of cell motility and epithelial morphogenesis by Jun kinases. Trends Cell Biol 14:94-101.

Xiao J, Liu Y (2003) Differential roles of ERK and JNK in early and late stages of neuritogenesis: a study in a novel PC12 model system. J Neurochem $86: 1516-1523$.

Yao R, Yoshihara M, Osada H (1997) Specific activation of a c-Jun NH2terminal kinase isoform and induction of neurite outgrowth in PC-12 cells by staurosporine. J Biol Chem 272:18261-18266.
Yoshida H, Hastie CJ, McLauchlan H, Cohen P, Goedert M (2004) Phosphorylation of microtubule-associated protein tau by isoforms of c-Jun N-terminal kinase (JNK). J Neurochem 90:352-358.

Yoshimura T, Kawano Y, Arimura N, Kawabata S, Kikuchi A, Kaibuchi K (2005) GSK-3beta regulates phosphorylation of CRMP-2 and neuronal polarity. Cell 120:137-149.

Yoshimura T, Arimura N, Kawano Y, Kawabata S, Wang S, Kaibuchi K (2006) Ras regulates neuronal polarity via the PI3-kinase/Akt/GSK3beta/CRMP-2 pathway. Biochem Biophys Res Commun 340:62-68.

Zheng C, Xiang J, Hunter T, Lin A (1999) The JNKK2-JNK1 fusion protein acts as a constitutively active c-Jun kinase that stimulates c-Jun transcription activity. J Biol Chem 274:28966-28971. 